\title{
Understanding Stakeholder Interactions Impacting Human Spaceflight Funding Levels
}

\author{
Brian M. Gardner ${ }^{(凶)}$ and Mo Mansouri \\ School of Systems and Enterprises, Stevens Institute of Technology, \\ 525 River Street, Hoboken, NJ 07030, USA \\ \{bgardne1, mmansour\} astevens. edu
}

\begin{abstract}
Human space exploration is an important endeavor for mankind. Exploration helps us to better understand our environment, offers the possibilities of new resources and the development of technologies to benefit humanity. Due to the complex nature of human spaceflight, programs are generally funded by governments using public funds. This paper uses a systems thinking framework to identify the key stakeholders in the human spaceflight funding system of interest, illuminate the interrelationships between the stakeholder's interests and demonstrate stakeholder influence on human spaceflight funding levels. The systems thinking assessment of the human spaceflight funding system results in a Causal Loop Diagram which can be used as the starting point for a systems dynamic model.
\end{abstract}

